\title{
THE PAH HYPOTHESIS AND THE EXTINCTION CURVE
}

\author{
A. LÉger, L. Verstraete, L. D'Hendecourt, D. \\ DÉFOURNEAU, \\ Groupe de Physique des Solides de l'E.N.S., Université Paris VII, Tour 29 \\ 2, place Jussieu 75251 Paris Cédex 05 - France \\ O. Dutuit, \\ Physico-Chimie des Rayonnements, Bâtiment 950, Université Paris XI \\ F91405 ORSAY - France \\ W. SCHMIDT, \\ Biochemical Institute, Sieker Landstrasser 19, 2070 AHRENSBURG- FRG \\ J. C. LAUER \\ Centre de Recherches Lorraine, NORSOLOR, Usine de Marienau \\ 57612 FORBACH - France
}

\section{THE PAH HYPOTHESIS}

The Polycyclic Aromatic Hydrocarbon (PAH) hypothesis states that a mixture of free PAH molecules is an ubiquitous and abundant component of the interstellar matter. It has been first formulated by Léger and Puget (1984) on the basis of molecular stability that made PAHs good candidates for the very small grains proposed by Sellgren (1984). Then, they obtained strong, although not final, support from spectroscopy.

\section{INTRINSIC DIF FICULTIES TO MAKE A PRECISE SPECTROSCOPIC IDEN- TIFICATION}

They arise because:

(i) dealing with a mixture of numerous species (see Fig. 1), one cannot expect a sharp spectroscopic fit between the lines of one molecule and the observations such as obtained between the rotational lines of methanol and the corresponding features in radio data. Fortunately, IR spectra have bands that depend almost only on atomic groups and are common to whole families of species (compact PAHs in our case). One cannot expect however a complete agreement unless the exact composition of the mixture is known; 
TABLE 1

INTERSTELLAR CONDITIONS COMPARED WITH LABORATORY CONDITIONS

\begin{tabular}{ll}
\hline \multicolumn{1}{c}{ Interstellar } & \multicolumn{1}{c}{ Laboratory } \\
\hline \hline Isolated species & $\begin{array}{l}\text { Molecular solids (most data) } \\
\text { Isolated (few data- gas phase } \\
\text { and rare gas matrix) }\end{array}$ \\
\hline $\begin{array}{l}\text { Ionized (expected in } \\
\text { most present observations) }\end{array}$ & Neutral \\
\hline Partially hydrogenated & Fully hydrogenated \\
$\left(\alpha_{H} \sim 30 \%\right)$ & $\left.\alpha_{H}=100 \%\right)$ \\
\hline & $300 \mathrm{~K}$ (mostly) \\
Temperatures: & $4-70 \mathrm{~K}$ (matrices) \\
& $500 \mathrm{~K}$ (gas phase) \\
\hline & Absorption + calculation \\
Emission & Emission: experiment by \\
& Cherchneff and Barker (1989)
\end{tabular}

(ii) present laboratory analogues of interstellar (IS) PAHs are only approximate. The situation is schematically summarized in Table 1.

\section{SPECTROSCOPIC COMPARISON (IR)}

From the work of Duley and Williams (1981), Léger and Puget (1984), Allamandola, Tielens and Barker (1985) and Léger, d'Hendecourt and Défourneau $(1987,1988 b)$, it can be concluded that:

(i) a mixture of compact PAHs can give a reasonably good fit to the whole set of observed features but the $11.3 \mu \mathrm{m}$ one (see Fig. 11 in Léger et al., 1988b);

(ii) domination of the $11.3 \mu \mathrm{m}$ band over the 12 and $13 \mu \mathrm{m}$ ones can be understood (dehydrogenation);

(iii) other differences reported in Table 1 do not seem to upset the spectra;

(iv) emission mechanism is understood.

Several actual predictions of the PAH hypothesis have already been observed:

(i) mid-IR emission of Cirrus (predicted by Puget et al., 1985 - observed by Boulanger et al., 1985); 


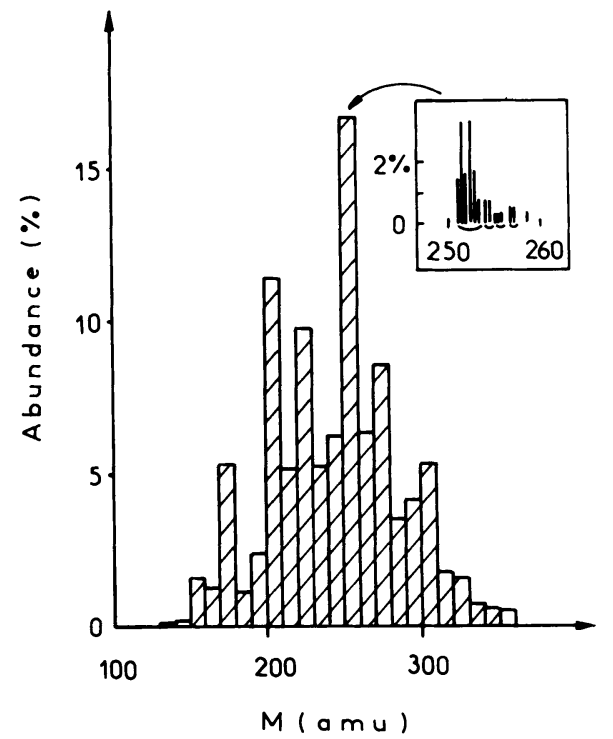

Fig. 1. Mass histogram of the coal pitch extract. Components are gathered in $10 \mathrm{amu}$ bins. The composition of one of them is shown in detail.

(ii) ubiquity of the $3.3 \mu \mathrm{m}$ feature in the diffuse emission of the Galaxy (predicted by Puget et al., 1985 - observed by the AROME experiment: Giard et al., 1988, 1989);

(iii) presence of an emission band at $5.3 \mu \mathrm{m}$ (predicted by Léger and d'Hendecourt, 1987 - observed by Allamandola et al., 1989).

Other implications can be searched for in the future:

(i) presence of a (weak) $1.68 \mu \mathrm{m}$ band, harmonic of the $3.28 \mu \mathrm{m} \mathrm{CH}$ mode;

(ii) $12 / 11.3 \mu \mathrm{m}$ relative importance in cirrus compared to bright reflection nebulae;

(iii) $158 \mu \mathrm{m} C \mathrm{II} / \mathrm{PAH}$ bands correlation if the IS gas heating is due to the photoelectric effect on PAHs.

\section{VISUAL ABSORPTION AND RED FLUORESCENCE}

PAH ions have middle to strong absorptions $\left(\mathrm{f} \sim 10^{-1}\right)$ in the visual and are proposed as candidates for the DIB carriers [van der Zwet and Allamandola, 1985; Léger and d'Hendecourt, 1985; Crawford et al., 1985]. Fluorescence processes in PAHs, including Poincaré fluorescence (Léger et al., 1988a), are proposed for the red fluorescence observed in reflection nebulae and high latitude HI clouds (Ryter and d'Hendecourt, 1989). 


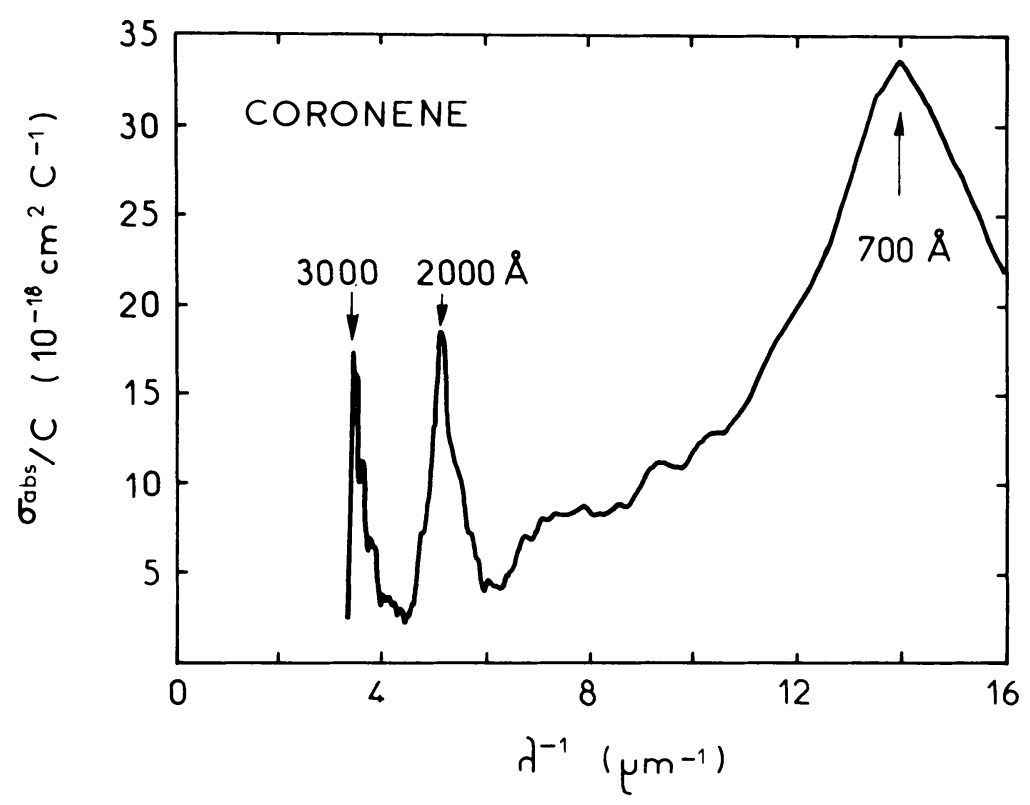

Fig. 2. Absorption cross section of coronene per carbon atom.

\section{UV ABSORPTION OF PAH MIXTURES}

The PAH hypothesis implies that they absorb in the visual-UV the substantial fraction of the energy that they re-emit in mid-IR. The model by Puget et al. (1985) of the IS matter attributes the IRAS $12 \mu \mathrm{m}$ band diffuse emission to PAHs - in fact, it had predicted it. It represents typically $18 \%$ of the total IRAS flux (Boulanger and Pérault, 1988). The $25 \mu \mathrm{m}$ band emission and about $2 / 3$ of the 60 $\mu \mathrm{m}$ one are also attributed to fluctuating particles (Ryter et al., 1988). The lack of spectroscopical information prevents their precise identification and this situation may last in the future. However, natural candidates are (big) PAHs. They represent $24 \%$ of the total.

Thus, PAHs would be responsible for $18 \%$ and possibly $42 \%$ of the absorption by the IS matter. A question arises: in what part of the extinction curve does it occur? And is there a conflict between the sharp bands of individual PAHs in the UV (Donn, 1968) and their absence in the observations?

We have undertaken UV measurements on coronene and different natural mixtures of PAHs, using the Orsay synchrotron radiation facility (LURE). Mixtures of PAHs are produced by subliming a coal pitch extract whose composition is determined by mass spectroscopy and chromatography (Fig. 1). Due to the high temperature (1400JK) during pitch manufacture, it contains only aromatic species. Over $\mathbf{3 0 0}$ molecules are identified in this PAH mixture and the most abundant ones are only at the level of $3 \%$.

The IR spectrum exhibits sharp $3.3-6.2-11.3-12.0-13.5 \mu \mathrm{m}$ and broad 
7.7 $\mu \mathrm{m}$ bands. The extract is vaporized under vacuum in an oven with pinholes for the UV beam and an absolute pressure gauge. A finite sample being vaporized, the vapor composition varies with time as the oven temperature is gradually increased to maintain a constant pressure. This gives us the possibility of studying different mixtures.

The UV cross sections of coronene and PAH mixtures are reported in Fig. 2 and 3. The near UV part of the coronene spectrum agrees with previous measurements in solution (UV Atlas, 1966) but, for the first time, its far-UV absorption is obtained. It exhibits a strong peak at $700 \AA$ which is also present in benzene and graphite and could be common to all aromatic species.

The absorption curves of PAH mixtures are presently measured only for $\lambda^{-1}<$ $7.7 \mu \mathrm{m}^{-1}$. They all show:

- little absorption for $\lambda>4000 \AA, \lambda^{-1}<2.5 \mu \mathrm{m}^{-1}$

- band gathering in [2500 - $1800 \AA],\left[4-5.6 \mu \mathrm{m}^{-1}\right]$

- depression around $1500 \AA, 6.7 \mu \mathrm{m}^{-1}$

- FUV increase for $\lambda<1500 \AA, \lambda^{-1}>6.7 \mu \mathrm{m}^{-1}$.

They show a strong similarity with the UV part of the IS extinction curve but for the width of the $2200 \AA$ bump. Does this latter point only indicate that we have not used a PAH mixture close enough to the interstellar one $e^{1}$ or is it more fundamental?

\section{PAH CONTRIBUTION TO THE EXTINCTION CURVE}

Using the above absorption as a guide, let us derive the contribution of PAHs to the IS Absorption Curve from the energy budget. We adopt the analysis of the extinction curve by Greenberg and Chlewicki (1983) that distinguishes: (1) a large grain component and (2) a small "grain" component which is purely absorbing and produces the $2200 \AA$ bump and the far-UV rise. Using a plausible albedo for large grains and a zero albedo for component (2), we can derive an interstellar absorption curve and a global albedo (Fig. 4). The latter agrees well with the values derived by Lillie and Witt (1976) from their study of the diffuse Galactic light. The comparison of Fig. 3 and 4 indicates that PAHs likely contribute to component (2). To what level? The energy budget will give us a strong constraint.

Fig. 5 shows the Interstellar Radiation Field $U_{n} u$ and the power absorbed by the IS matter as:

$$
P_{\nu}=C U_{\nu} \tau_{\nu}^{a b s}
$$

Component (2) is found to absorb about $40 \%$ of the total. Now, if PAHs emit and therefore absorb $18 \%$, or $42 \%$, of the total power, they must be responsible for half, or all, of the component (2) contribution.

\footnotetext{
${ }^{1}$ For instance, the mass distribution of Fig. 1 is centered at 250 amu whereas the interstellar species are expected to be larger $(600 \mathrm{amu})$, Léger et al., $1988 \mathrm{~b}$.
} 


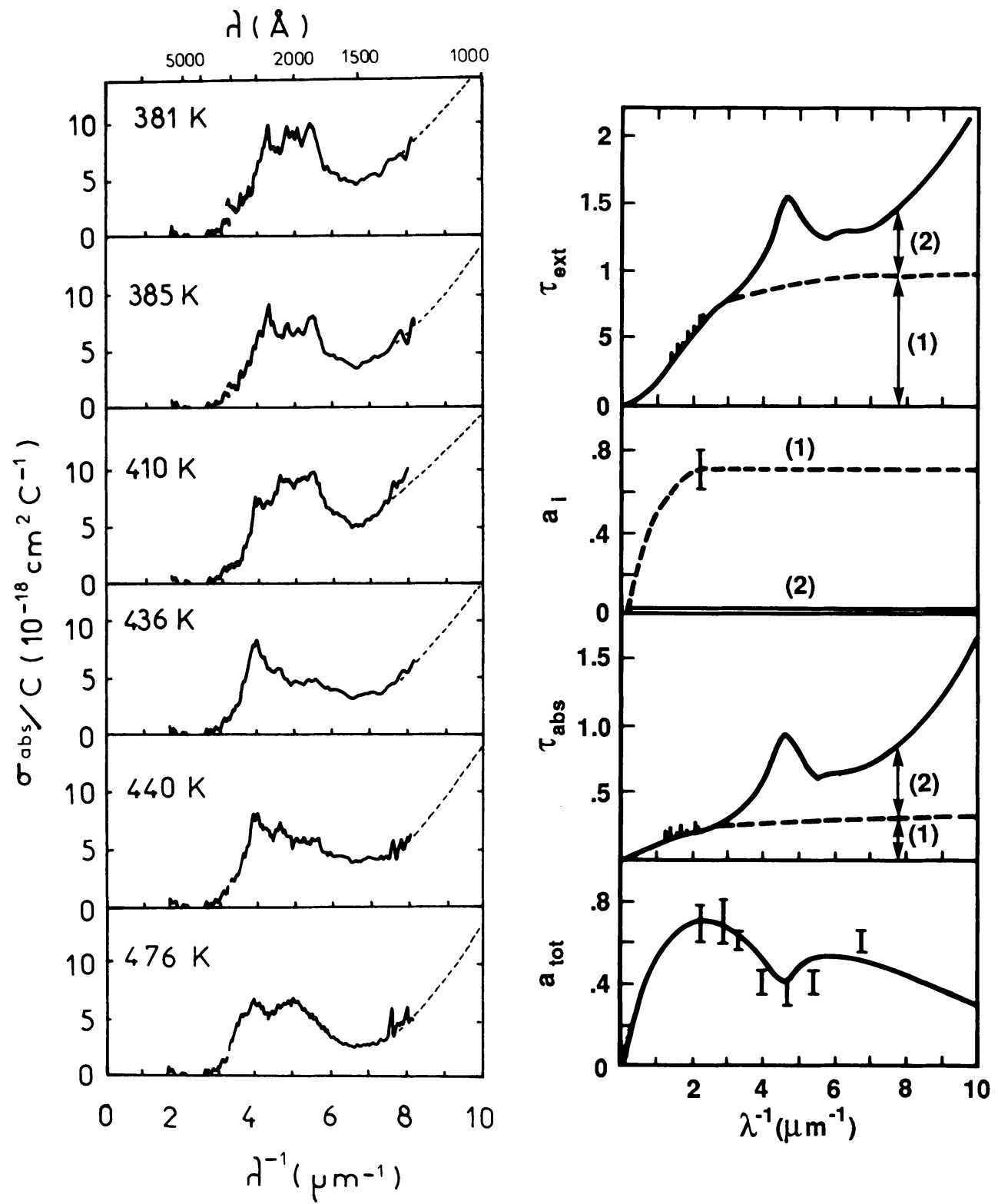

Fig. 3. Left: Absorption cross sections of different PAH mixtures (full line) and coronene (dotted line). The temperature of the oven is reported that determines the mixture as explained in the text. Fig. 4. Right: Extinction curve $r^{\text {ext }} /\left(10^{-21} \mathrm{~cm}^{2} \mathrm{H}^{-1}\right)$ analyzed in components (1) (large grains) and (2) with assumed albedos $a_{1}$ and $a_{2}$ respectively. Resulting absorption curve $\tau^{a b s} /\left(10^{-21} \mathrm{~cm}^{2} \mathrm{H}^{-1}\right)$ and total albedo. The points with errors bars are values derived by Lillie and Witt (1976) from their observations. 


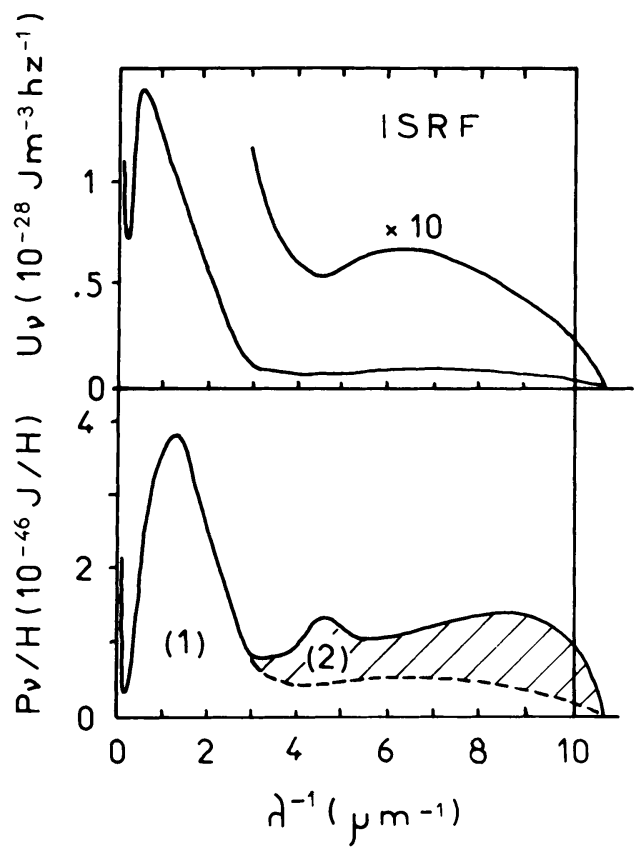

Fig. 5. ISRF spectrum and resulting absorbed spectral power, $P_{\nu / H}$, as a function of $\lambda^{-1}$. Contributions of components (1) and (2) are shown separately. The absorbed energy per hydrogen, $\int P_{\nu / H} d \nu$, is found $4.8 \times 10^{-31} \mathrm{~W} / H$, in reasonable agreement with Mathis et al., 1983$)\left(4.2 \times 10^{-31}\right.$ $\mathrm{W} / H)$ and Puget, $1988\left(5.7 \times 10^{-31} \mathrm{~W} / H\right)$.

\section{IONIZATION CROSS SECTION OF PAHS}

The fraction of the absorption cross section which leads to ionization has been measured separately and the implications for the heating of the IS gas by photoelectrons derived. It is reported at this conference by Verstraete et al., 1989.

\section{CONCLUSIONS}

- The similarity between the UV absorption cross sections of the PAH mixtures measured and the IS absorption curve cannot be considered as a spectroscopic identification because bands are too broad.

- However, if the PAH identification in the IR is accepted, the UV data make a coherent picture as opposed to what the inspection of the spectrum of an individual single PAH spectra may have suggested.

- The energy balance directly implies that PAH molecules are responsible for a significant fraction ( $50 \%$ ), or most, of the $2200 \AA$ bump plus FUV extinction.

- There is a need for performing FUV $(\lambda<1500 \AA)$ measurements on PAH mixtures to check whether they show a correlation between the $2200 \AA$ bump and the FUV rise. There is also need for measurement on other (heavier?) 
and the FUV rise. There is also need for measurement on other (heavier?) mixtures to check whether the disagreement with the width of the IS $2200 \mathrm{~J} \AA$ bump remains.

- The present interpretation of the UV curve predicts a decreasing albedo at wavelengths shorter than $1450 \AA$.

\section{REFERENCES}

Allamandola, L. J., Tielens, A. G. G. M., and Barker, J. R. 1985, Ap. J. (Letters), 290, L25.

Allamandola, L. J. 1989, in IAU Symposium 195, Interstellar Dust, eds. L. J. Allamandola and A. G. G. M. Tielens, (Dordrecht: Kluwer), p. 129.

Boulanger, F. and Pérault M. 1988, Ap. J., 330, 964.

Boulanger, F., Baud B., van Albada, G. D. 1985, Astr. Ap., 144, L9.

Cherchneff, I., and Barker, J. 1989, in Interstellar Dust Contributed Papers, eds. A. G. G. M. Tielens and L. J. Allamandola, NASA CP-3036.

Crawford, M. K., Tielens, A. G. G. M., Allamandola, L. J. 1985, Ap. J. (Letters), 293 , L45.

Donn, B. 1968, Ap. J. (Letters), 152, L129.

Duley, W. W. and Williams, D. A. 1981, M. N. R. A. S., 196, 269.

Giard, M., Pajot, F., Lamarre, J. M., Serra, G., Andouze, J., Caux, E., Combes, M., Gispert,JR., Léger, A. and Rouan, D. 1988, Astr. Ap., 201, L1.

Giard, M., Pajot, F., Caux, E., Lamarre, J. M., and Serra, G. 1989, in Interstellar Dust Contributed Papers, eds. A. G. G. M. Tielens and L. J. Allamandola, NASA CP-3036.

Greenberg, M. and Chlewicki, G. 1983, Ap. J., 272, 563.

Léger, A. and d'Hendecourt, L. 1985, Astr. Ap., 146, 81.

-. 1987, in Polycyclic Aromatic Hydrocarbons and Astrophysics, eds. Léger, A., d'Hendecourt, L., and Boccara, N., (Dordrecht: Reidel), p. 223.

Léger, A. and Puget, J. L. 1984, Astr. Ap., 137, L5.

Léger, A., Boissel, P. and d'Hendecourt, L. 1988a, Phys. Rev. Letters, 60, 921.

Léger, A., d'Hendecourt, L. and Défourneau, D., $1988 b$, Astr. Ap., in press.

Lillie, C. F. and Witt, A. N. 1976, Ap. J., 208, 64.

Mathis, J. S., Metzger, P. G. and Panagia, N. 1983, Astr. Ap., 128, 212.

Puget, J. L. 1988, in Comet to Cosmology, ed. Lawrence, (Springer-Verlag), p. 118.

Puget, J. L., Léger, A. and Boulanger, F. 1985, Astr. Ap., 142, L19.

Ryter, C., Puget, J. L. and Pérault, M. 1988, Astr. Ap., 186, 312.

Ryter, C., and d'Hendecourt, L. 1989, in Interstellar Dust Contributed Papers, eds. A. G. G. M. Tielens and L. J. Allamandola, NASA CP-3036.

Sellgren, K. 1984, Ap. J., $277,623$.

UV Atlas 1966, (London: Butterworths).

van der Zwet, G. P. and Allamandola, L. J. 1985, Astr. Ap., 146, 77.

Verstraete, L., Léger, A., d'Hendecourt, L., Dutuit, O., Défourneau, D. 1989, in Interstellar Dust Contributed Papers, eds. A. G. G. M. Tielens and L. J. Allamandola, NASA CP-3036. 